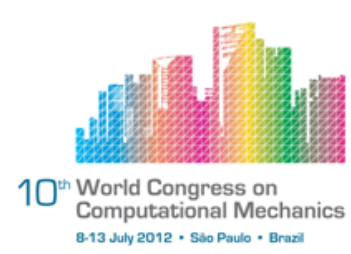

\title{
OPTIMUM PRE-STRESS DESIGN FOR FREQUENCY REQUIREMENT OF TENSEGRITY STRUCTURES
}

\author{
Seif Dalil Safaei, Anders Eriksson, Gunnar Tibert
}

KTH Mechanics Royal Institute of Technology, Osquars backe 18, SE-100 44 Stockholm, Sweden

\begin{abstract}
Structures composed of tension and compression elements in equilibrium are denoted tensegrity structures. Stability of tensegrity structures is achieved through introducing initial member forces (pre-stress). The pre-stress design can be seen consisting of three different stages: (i) finding the bases of possible pre-stress states, (ii) finding admissible distributions considering unilateral properties of the elements and stability of the structure, (iii) finding the optimum pre-stress pattern for certain magnitude from compatible pre-stress states. So far, no research has been carried out to connect the three steps, i.e. finding a suitable prestress pattern which also considers mechanical properties of the highly pre-stressed structure e.g. its natural frequencies. This paper aims at finding an optimum pre-stress pattern and level of pre-stress for the maximum frequency. The pre-stress problem is on a linear static level where no slackening is allowed. An optimization is performed to find the optimum prestress pattern from the self-stress modes obtained by a singular value decomposition (SVD) of the equilibrium matrix. The objective function is the first natural frequency of the structure. Finite element analysis is employed for the linear analysis of the structure and a genetic algorithm for optimization i.e., a non-gradient method. The example considered is a double layer tensegrity grid consisting of 29 independent self-stress states. The method is applicable to complex asymmetric three-dimensional structures. The new aspect of this work is a link between the SVD analysis, finite element analysis and genetic algorithm.
\end{abstract}

Keywords: Tensegrity structures, Pre-stress, Finite element analysis, Genetic algorithm.

\section{Introduction}

Structures composed of tension elements (strings, tendons or cables) and compression elements (bars or struts) are often denoted tensegrity structures [1]. Distribution of member forces at the self-equilibrium state, i.e., pre-stresses introduced into the members, greatly contributes to the stiffness and stability of tensegrity structures. The process of determining member forces for the structure with a given shape is called initial force or initial pre-stress design. The pre-stresses should be assigned considering the stress unilateral property of the members; i.e., cables and struts must be under tension and compression, respectively. To the author's knowledge, no research have been carried out for determination of the pre-stress 
distribution to (i) stabilize the structure, (ii) satisfy some some structural properties and (iii) consider unilateral properties of the cables and struts.

On the research of pre-stress finding Quirant [2] expands the Pellegrino method [3] to tensegrity structures by considering the unilateral behavior of the cable elements. Tran and Lee [4] present a method for finding a single self-stress mode taking into account the unilateral member properties and the stability of the structure. Xu and Luo [5] use a simulated annealing algorithm to solve an optimization model for the pre-stress design. Connelly [6] introduces a stability criterion called super stability, and Ohsaki and Zhang [7] study stability conditions of pre-stressed pin jointed structures and find that a pre-stressed structure can be unstable if the pre-stress is moderately large.

A genetic algorithm (GA) is a non-gradient optimization method frequently employed to address various problems of tensegrity structures. It is interesting to mention that, the main contribution of GA for tensegrities was for form-finding, [9-14]. El-Lishani et al. [8] employed GA to find the stability characteristics of simultaneously statically and kinematically indeterminate structures.

\section{Problem formulations}

An infinite number of independent admissible self-stress combinations could be found for a tensegrity structure with more than one state of self-stress, by superposing linear combinations of the base states. The following assumptions are considered in this study:

- The form-finding procedure is performed and the nodal coordinates and their connectivity is known.

- No external load is applied on the structure and the self-weight is not considered during the pre-stress design.

\subsection{Basic concepts of pre-stress}

Pellegrino [3] uses the Singular Value Decomposition (SVD) of the equilibrium matrix $\mathbf{H}$ to obtain the mechanism modes for a three-dimensional pin-jointed framework with $j$ joints, $b$ bars and $c$ kinematic constraints:

$$
\mathbf{H}=\mathbf{U} \boldsymbol{\Sigma} \mathbf{W}^{T}
$$

where $\mathbf{H}$ is the $3 j-c \times b$ equilibrium matrix, $\mathbf{U}$ is a $3 j-c \times 3 j-c$ orthogonal matrix, $\mathbf{W}$ is a $b \times b$ orthogonal matrix, and $\Sigma$ is a $3 j-c \times b$ matrix with non-negative elements on the leading diagonal and all other elements zero. Once the rank of equilibrium matrix $r_{\mathrm{H}}$ has been found the number of states of self-stress and mechanism are

$$
\begin{gathered}
s=b-r_{\mathrm{H}} \\
m=3 j-c-r_{\mathrm{H}}
\end{gathered}
$$

Base for the mechanisms and states of self-stress are given by 


$$
\begin{aligned}
& \mathbf{S}=\left[u_{1} u_{2} \ldots u_{r_{a}} \mid m_{1} \ldots m_{m}\right] \\
& \mathbf{W}=\left[w_{1} w_{2} \ldots w_{r_{a}} \mid g_{1} \ldots g_{s}\right]
\end{aligned}
$$

If $s=1$, there is only one vector $\mathbf{g}_{1}$ which naturally satisfies the unilateral properties and $\mathbf{H g}_{1}=\mathbf{0}$. Otherwise, $s>1$, a linear admissible combination of $s$ independent states of self-stress mode should be computed as the null space of the equilibrium matrix $\mathbf{H}$ cannot be employed directly (the equilibrium matrix does not have anything to do with properties of members). A feasible state of self-stress can be represented as:

$$
\mathbf{p}_{f}=\mathbf{G c}
$$

where $\mathbf{p}_{f}$ is a $b \times 1$ feasible states of self-stress. This paper is all about computing a vector $\mathbf{c}=\left(c_{1}, c_{2}, \ldots, c_{s}\right)^{T}$ which contains the coefficients of $s$ independent self-stress states. The vector $\mathbf{G}, b \times s$, is the $s$ independent self-stress states. The coefficients should be selected in a way so that unilateral properties of the members, stability and frequency criteria of the structure fulfilled.

\subsection{Optimization formulation}

- Objective function: The objective to increase the first natural frequency of the structure is formulated as:

Minimize: $1 / f_{1}$

where $f_{1}$ is the first natural frequency of the structure in a pre-stress state.

- Constraints: The unilateral property and stability of the structure are the only constraints. The cable and bar elements should have positive and negative coefficients respectively, in the vector of self-stress. If the tangent stiffness matrix with these member forces introduced is positive definite, i.e, its six rigid body motion restrained, then the structure is initially stable.

- Variables: The coefficient vector c.

\section{Pre-stress design}

\subsection{Procedure}

Figure 1 shows the flowchart of the proposed method for initial pre-stress design. The procedure of finding an optimum pre-stress pattern is:

- The form-finding process is performed and the topology and nodal coordinated are determined.

- The Equilibrium matrix is computed by a method from Pellegrino [3]. The SVD analysis of equilibrium matrix gives the basis of the pre-stress. 
- A random number of individuals is produced. The individuals are the coefficients of $s$ independent self-stress state vectors $\mathbf{c}$.

- The self-stress vector $\mathbf{p}$ is computed and then, the unilateral properties and the stability of the structure are evaluated. Individuals which do not satisfy these conditions give singular tangent stiffness matrix, and they should not go to the FEM program. A very large fitness value far from the optimum one is assigned to these individuals. So they have very low chance of contribution to the next generations.

- The finite element analysis determines the first natural frequency and GA operators could lead the individuals to the optimum one. Table 1 shows the initial setup of the Matlab GA toolbox.

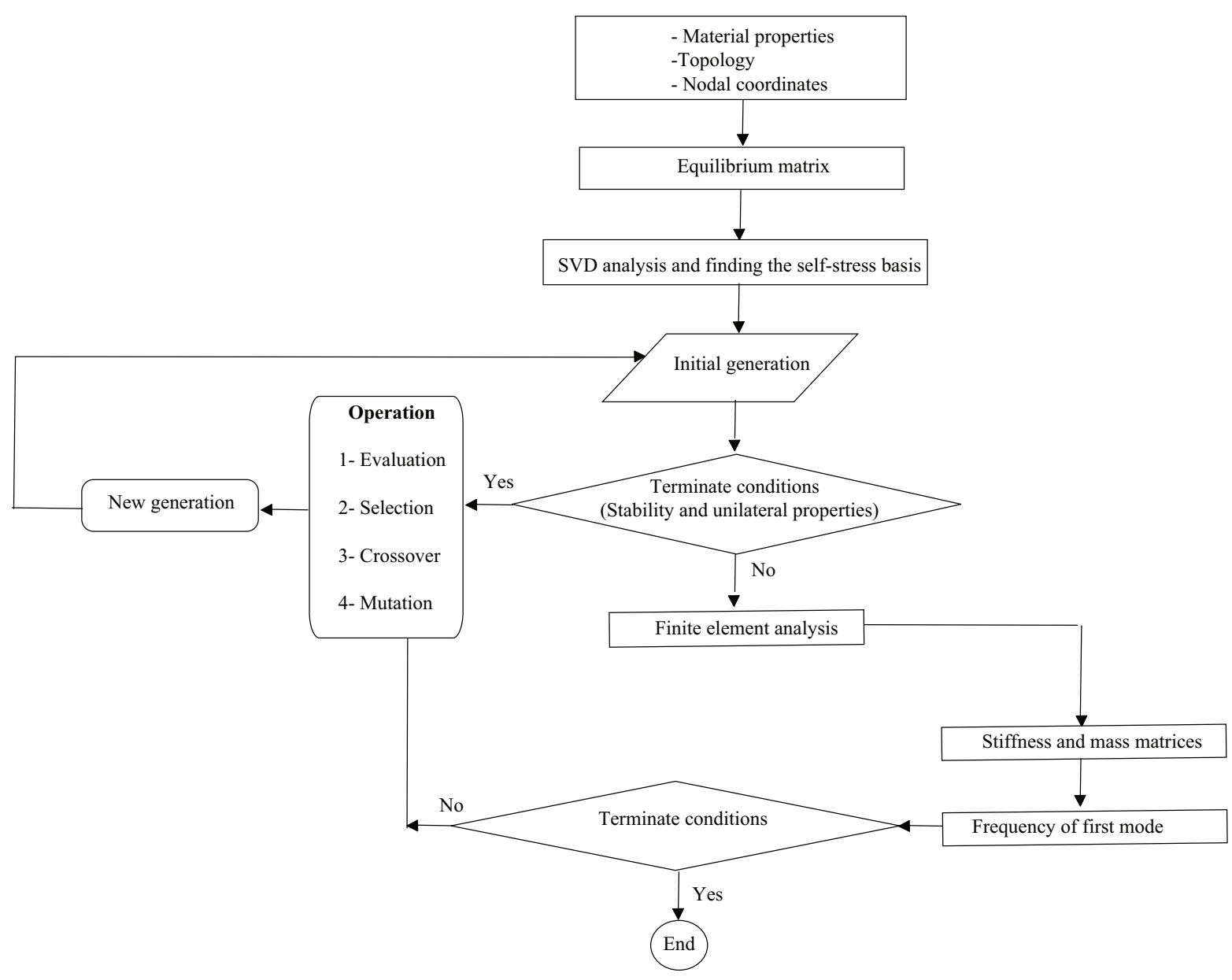

Figure 1. Flowchart of the proposed method.

\subsection{Pre-stress calculation}

The unique feature of this method is considering pre-stress level for finding the optimum self-stress pattern. The pre-stress related to each self-stress level is calculated as:

$$
\mathbf{p}=P R \boldsymbol{\alpha}
$$


where $\boldsymbol{\alpha}$ is an admissible self-stress vector $\mathbf{p}_{f}$ of length 1 . It is assumed that the total summation of all member forces is constant for all pre-stress patterns. A correction coefficient $R$ is employed to keep the total element forces constant for all cases. The total element forces of self-stress vector $\boldsymbol{\alpha}$ is compared with the symmetric case (elements in the similar position have the same force), and $P$ is the pre-stress levels determined by the designer.

\subsection{Tangent stiffness matrix formulation}

For the non-linear equilibrium analysis in this study, the bar element stiffness formulation by Guest [9] was used. The tangent stiffness matrix $\mathbf{K}$ relates the displacement increment $\delta \mathbf{d}$ at each node in all degrees of freedom to increments in the applied load $\delta \mathbf{e}$ as:

$$
\mathbf{K} \delta \mathbf{d}=\delta \mathbf{e}
$$

The tangent stiffness matrix $\mathbf{K}$ is dependent on topology, geometry, axial stiffness of the elements and element forces in the following form:

$$
\mathbf{K}=\mathbf{H} \widehat{\mathbf{G}} \mathbf{H}^{T}+\mathbf{S}
$$

where $\mathbf{H}$ is the equilibrium matrix and $\widehat{\mathbf{G}}$ is a diagonal matrix of modified axial stiffnesses, with an entry for each member $i$

$$
\widehat{g_{i}}=g_{i}\left(1-\varepsilon_{i}\right)
$$

where $g_{i}=d t / d l$ is the axial stiffness and $\varepsilon_{i}$ is the strain for the member. The axial force in a member is $t$. The matrix $\mathbf{S}$ is the stress matrix for the structure, which is written as the Kronecker product of a small or reduced stress matrix and a 3-by-3 identity matrix I,

$$
\mathbf{S}=\mathbf{\Omega} \otimes \mathbf{I}
$$

and the coefficients of the small stress matrix are given by

$$
\Omega_{i j}= \begin{cases}-\hat{t}_{i, j}=-\hat{t}_{j, i}, & \text { if } i \neq j \\ \sum_{k \neq i} \hat{t}_{i k}, & \text { if } i=j \\ 0, & \text { if there is no connection between } i \text { and } j\end{cases}
$$

in this formulation, $\hat{t}_{j, i}$ is the tension coefficient $(t / l)$ in the member that runs between nodes $i$ and $j$. The internal quasi-static equilibrium of a loaded structure is seen as

$$
\mathbf{f}=\mathbf{f}_{i}-\mathbf{e}=\mathbf{0}
$$

when the residual forces $\mathbf{f}$ are the difference between internal forces $\mathbf{f}_{i}=\mathbf{f}_{i}(\mathbf{d})$ and the external forces p. The internal forces are dependent on the structural displacement $\mathbf{d}$, but also on geometry, sectional properties and chosen pre-stress forces in the members. The non-linear equilibrium equation for a particular load case is solved by Newton iterations, utilizing the tangent stiffness relation, [10]. 


\subsection{Linear dynamic analysis}

To understand the vibration behavior of the tensegrity structures, the investigation of the fundamental mode of vibration is sought. Modal analysis then is performed with respect of changing different parameters. A linearized dynamic model of the structure is written as:

$$
\mathbf{M u ̈}+\mathbf{C} \dot{\mathbf{u}}+\mathbf{K u}=\mathbf{e}
$$

where $\mathbf{M}, \mathbf{K}, \mathbf{C}, \mathbf{p}$ and are the mass, damping, tangent stiffness matrices, and the external force vector, respectively. Also $\ddot{\mathbf{u}}, \dot{\mathbf{u}}$, and $\mathbf{u}$ are acceleration, velocity and displacement vectors, respectively. The modal analysis is conducted by ignoring the damping term and the vector of external force. Assuming a small harmonic motion of the form $\mathbf{u}=\overline{\mathbf{u}} \sin (\omega t)$, with $\omega$ as the frequency of system and $\overline{\mathbf{u}}$ as the amplitude vector:

$$
\mathbf{K} \overline{\mathbf{u}}=\omega^{2} \mathbf{M} \overline{\mathbf{u}}
$$

The generalized eigenvalue analysis for the matrices $\mathbf{M}$ and $\mathbf{K}$ gives the natural modes of the vibration. The mass matrix for each element $\mathbf{M}_{e}$ is computed as:

$$
\mathbf{M}_{e}=\frac{m}{6}\left[\begin{array}{cc}
2 \mathbf{I}_{3} & -\mathbf{I}_{3} \\
-\mathbf{I}_{3} & 2 \mathbf{I}_{3}
\end{array}\right]
$$

where $\mathbf{I}_{3}$ is a unit matrix and $m$ the mass of the element.

\subsection{Genetic algorithm for minimization}

The main parameters employed for the implementation of the genetic algorithm are presented in Table 1. The parameters are selected after a number of experiments, but there is no claim about their optimality. A sensitivity analysis should be performed to determine a set of optimal genetic algorithm parameters, including population size, crossover and mutation rates, etc. In general, the parameter set used in the experiments produces good-quality solutions in reasonable time.

Table 1. Main parameters of the genetic algorithm

\begin{tabular}{|l|l|}
\hline Parameter name & Type or value \\
\hline Population (type) & Double vector \\
Population(size) & 100 \\
Selection (function) & Stochastic uniform \\
Crossover (function) & Scattered \\
Mutation (function) & Use constant dependent default \\
Stopping criteria (generation number) & 100 \\
\hline
\end{tabular}

\section{Numerical examples}

The example considered here is a double layer tensegrity grid, Fig. 2. The structure consists of $12(4 \times 3)$ quadruplex modules, Fig. 3 . The quadruplex module has four struts and the self-stressed equilibrium is easily found from the rotation angle between the co-planar bases composed of cable polygons [11]. State of self-stress related to each single module is represented as: 
(a)

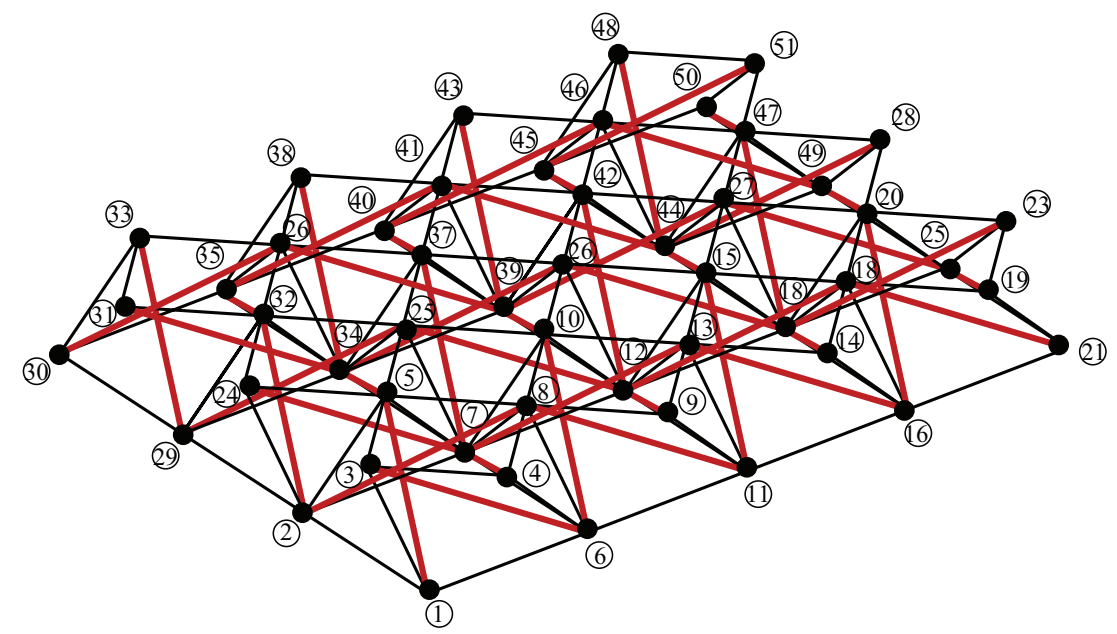

(b)

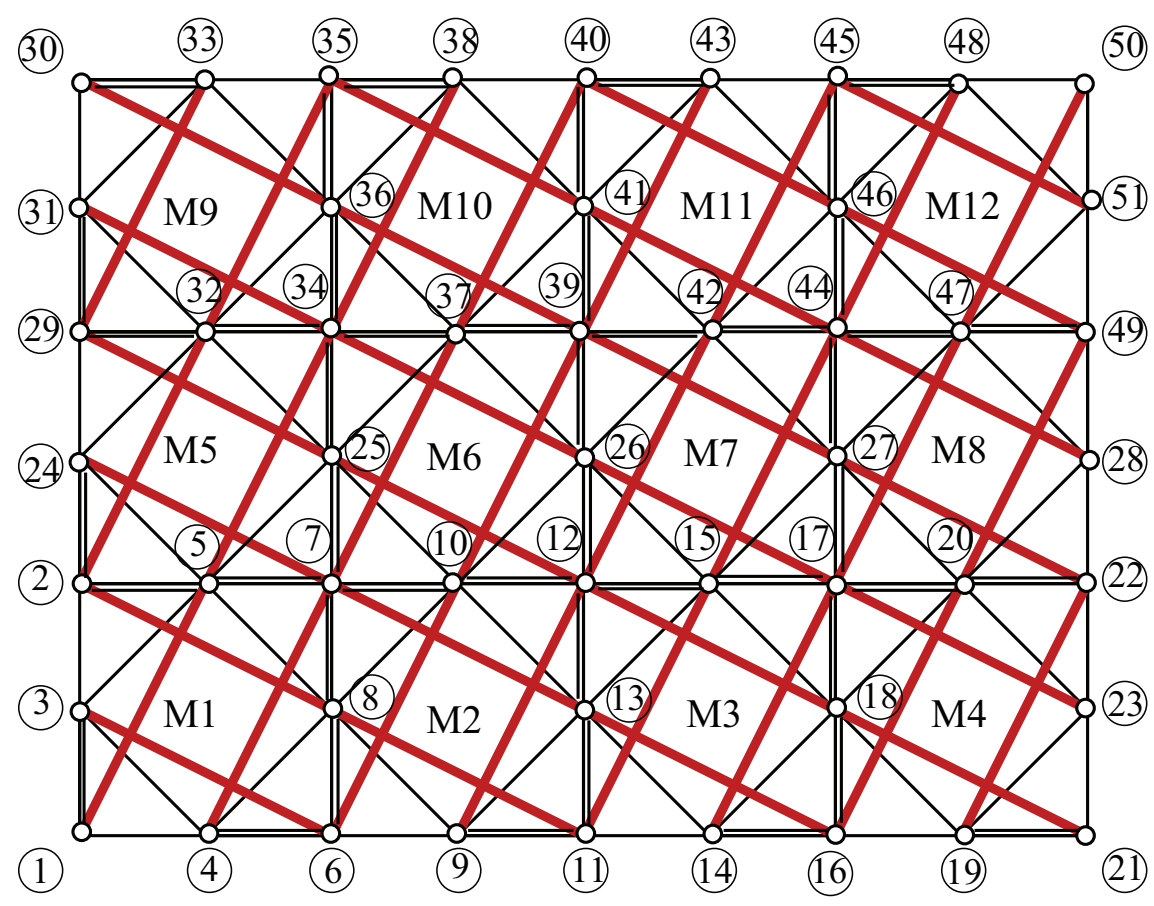

Figure 2. A three-dimensional tensegrity grid formed of $12(4 \times 3)$ quadruplex modules: (a) perspective view and (b) top view. $M$ is indicating the module number. 


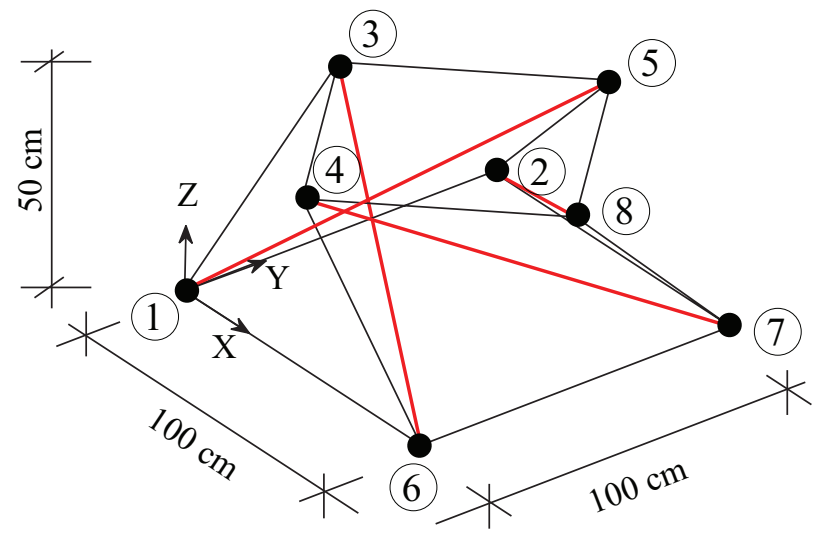

(a)

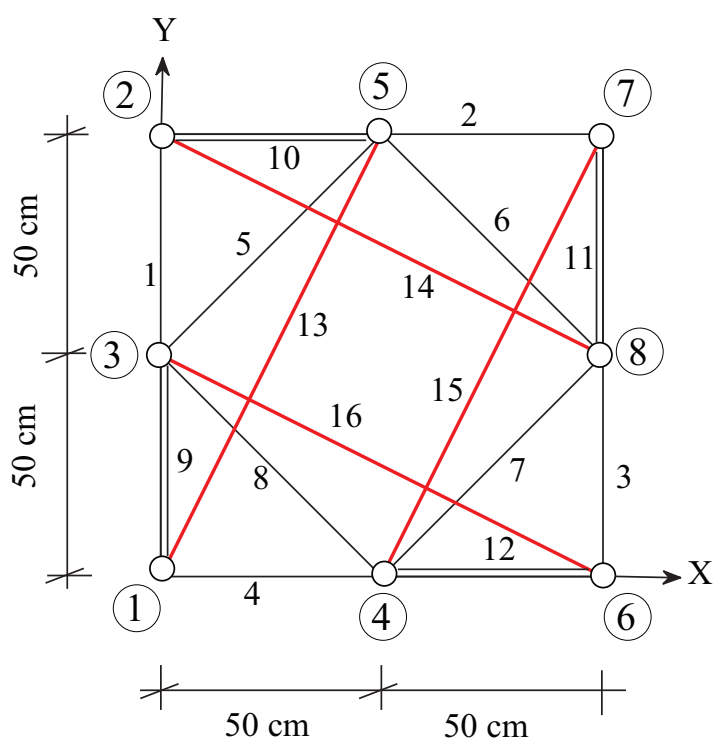

(b)

Figure 3. A unit quadruplex module: (a) perspective view and (b) top view.

$$
\begin{aligned}
& \boldsymbol{\alpha}_{\text {Base cables }}=(1,1,1,1) \\
& \boldsymbol{\alpha}_{\text {Side cables }}=\sqrt{2}(1,1,1,1) \\
& \boldsymbol{\alpha}_{\text {Top cables }}=\sqrt{2}(1,1,1,1) \\
& \boldsymbol{\alpha}_{\text {Bars }}=-\sqrt{6}(1,1,1,1) \\
& \boldsymbol{\alpha}_{\text {Quadruplex }}=\left(\boldsymbol{\alpha}_{\text {Base }}, \boldsymbol{\alpha}_{\text {Side }}, \boldsymbol{\alpha}_{\text {Top }}, \boldsymbol{\alpha}_{\text {Bars }}\right)
\end{aligned}
$$

The self-stress vector of the whole structure for a symmetric case is obtained by assembling the self-stress vector of each quadruplex module, and doubling the element shared between two modules. After determining the self-stress vector of the structure, each member of self-stress vector is divided by the norm of self-stress vector. The total summation of all member forces, $R$, is $2.79 \mathrm{~N}$. There exist 34 states of self-stress from an SVD analysis of the equilibrium matrix and no mechanisms when nodes 1,21, 30 and 50 are fully restrained. Tran and Lee [4] show that the force density matrix of this structure is positive semi-definite indicating that the structure is super stable regardless of the materials and the pre-stress levels.

The selected Young's modulus, density, and cross-section area of bar elements are $E=100 \mathrm{GPa}, \rho=1540 \mathrm{~kg} / \mathrm{m}^{3}$, and $A=2 \cdot 10^{-3} \mathrm{~m}^{2}$, respectively. The initial values for the cable elements are $E=130 \mathrm{GPa}, \rho=1840 \mathrm{~kg} / \mathrm{m}^{3}$, and $A=7.854 \cdot 10^{-5} \mathrm{~m}^{2}$, respectively. It is assumed that the elements do not confront buckling and yielding for the designed pre-stress levels.

Table 2 shows the optimum pre-stress patterns and their related first natural frequencies for different pre-stress levels. Each number in a coefficient column is the ratio of selfstress vector. For example, the self-stress vector of the structure of optimum pattern with $P=100 \mathrm{~N}$ is computed as 


$$
\boldsymbol{\alpha}_{\text {tensegrity grid }}=1.27 \cdot \boldsymbol{\alpha}_{\mathrm{M} 1}+0.63 \cdot \boldsymbol{\alpha}_{\mathrm{M} 2}+\ldots+1.27 \cdot \boldsymbol{\alpha}_{\mathrm{M} 12}
$$

Table 2 shows that the optimum pre-stress pattern is different for different pre-stress levels. This is naturally due to the non-linear relation of pre-stress level and the frequencies, Fig. 4.

Table 2. First natural frequencies of different pre-stress magnitudes.

\begin{tabular}{|c|c|c|c|c|c|}
\hline \multirow{2}{*}{ Pattern } & \multirow{2}{*}{ Coefficients c } & \multicolumn{4}{|c|}{$P(\mathrm{kN})$} \\
\hline & & 0.1 & 1 & 10 & 100 \\
\hline Symmetric pattern & $\begin{array}{llll}1.00 & 1.00 & 1.00 & 1.00 \\
1.00 & 1.00 & 1.00 & 1.00 \\
1.00 & 1.00 & 1.001 .00\end{array}$ & 0.7896 & 0.8659 & 1.3495 & 2.6756 \\
\hline Optimum pattern $P=0.1 \mathrm{kN}$ & $\begin{array}{llll}1.20 & 0.63 & 0.63 & 1.27 \\
1.27 & 0.63 & 0.63 & 1.27 \\
1.27 & 0.63 & 0.63 & 1.26\end{array}$ & 0.8150 & 0.8760 & 1.3964 & 2.5248 \\
\hline Optimum pattern $P=1 \mathrm{kN}$ & $\begin{array}{llll}1.26 & 0.63 & 0.64 & 1.27 \\
1.26 & 0.63 & 0.63 & 1.26 \\
1.27 & 0.64 & 0.64 & 1.27\end{array}$ & 0.7908 & 0.8890 & 1.3960 & 2.5268 \\
\hline Optimum pattern $P=10 \mathrm{kN}$ & 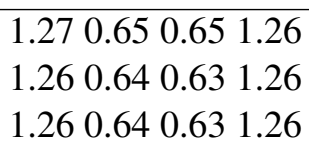 & 0.7908 & 0.8759 & 1.4157 & 2.5287 \\
\hline Optimum pattern $P=100 \mathrm{kN}$ & $\begin{array}{llll}0.78 & 1.35 & 1.35 & 0.68 \\
0.68 & 0.87 & 0.88 & 0.68 \\
0.68 & 1.35 & 1.35 & 0.79\end{array}$ & 0.7889 & 0.8601 & 1.3109 & 2.7433 \\
\hline
\end{tabular}

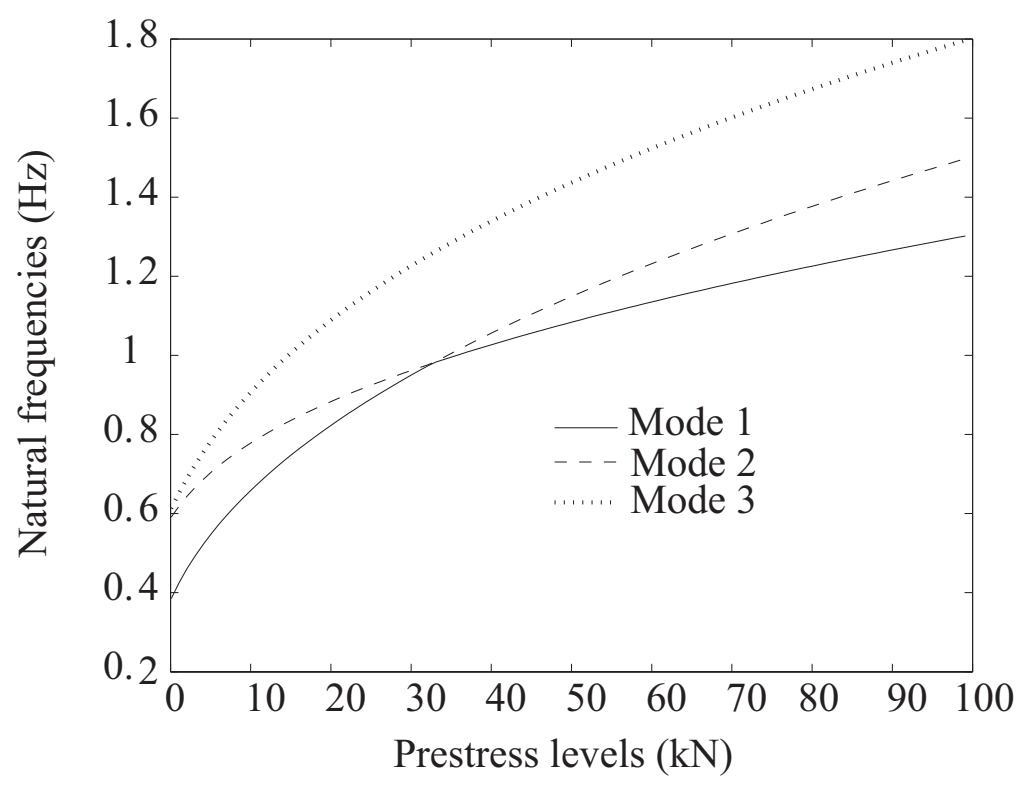

Figure 4. Natural frequencies of different pre-stress level for symmetric patterns (first result column in Table 2).

Figure 5 shows the convergence of GA. Best fitness and mean fitness are the smallest fitness value for any individual in the population and their average. Figure 5 shows an optimum close to the final optimum is obtained after 80 generations. 


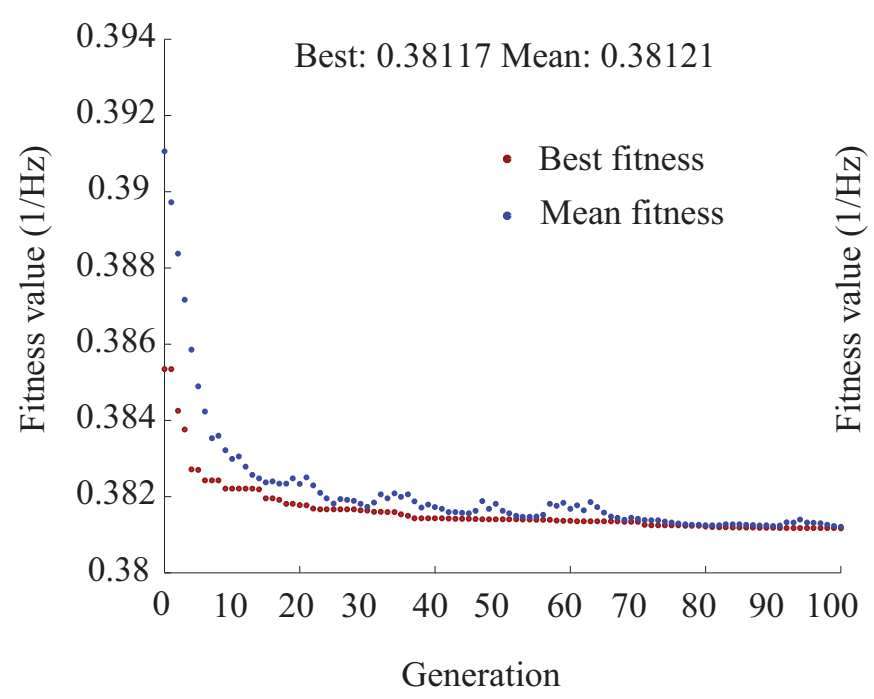

(a)

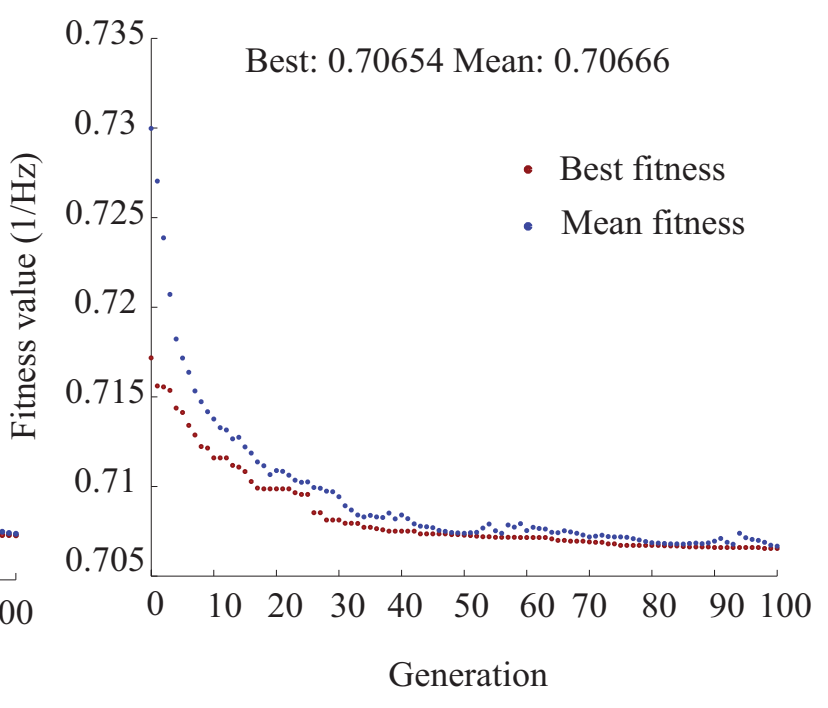

(b)

Figure 5. Best and mean fitness values versus number of generations for (a) $P=100 \mathrm{kN}$, and a) $P=10 \mathrm{kN}$.

An important question here is: does the initial material selection change the optimum pattern or not? To study this, various material are selected for struts , [12], and the influence of symmetric and optimum patterns are compared. Table 3 shows the first natural frequencies of the symmetric pattern and the optimum one for $P=100 \mathrm{kN}$. The result shows that the first natural frequency of the optimum pattern is always higher than that the symmetric one, and is independent of material properties.

Figures 6 and 7 show the first two mode shapes related to the two different pre-stress levels when node 1, 21, 30 and 50 are fully constrainted. It is clear that the level of prestress changes the mode shape. For example, the first and second modes for high and low pre-stress levels, respectively, are related to bending. Although the structure does not have any mechanism, the pre-stress level has an important influence on the mode shapes, $[13,14]$ . The final evaluation is related to study the influence of different pattern on the mode shapes of the structure. Several analyses show that the mode shape is not sensitive to the pre-stress pattern. 
Table 3. First natural frequencies for symmetric and optimum pattern $(P=100 \mathrm{kN})$.

\begin{tabular}{|l|l|l|l|l|}
\hline Material & $\begin{array}{l}\text { Density, } \\
\left(\mathrm{kg} / \mathrm{m}^{3}\right)\end{array}$ & $\begin{array}{l}\text { Modulus, } \\
(\mathrm{GPa})\end{array}$ & $\begin{array}{l}\text { Symmetric } \\
\text { pattern } \\
(\mathrm{Hz})\end{array}$ & $\begin{array}{l}\text { Optimum } \\
\text { pattern } \\
(\mathrm{Hz})\end{array}$ \\
\hline $\begin{array}{l}\text { Uni Mitsubishi } \\
\text { K13C2U UHM } \\
\text { fibers and epoxy } \\
\text { matrix }\end{array}$ & 1840 & 536 & 2.4440 & 2.5058 \\
\hline $\begin{array}{l}\text { Uni Torayca } \\
\text { M60J fibers with } \\
\text { epoxy }\end{array}$ & 1682 & 354 & 2.5561 & 2.6208 \\
\hline $\begin{array}{l}\text { Uni IM7 fibers } \\
\text { with epoxy ma- } \\
\text { trix }\end{array}$ & 1588 & 167 & 2.6306 & 2.6972 \\
\hline $\begin{array}{l}\text { Brush Wellman } \\
\text { AlBeMet }\end{array}$ & 2071 & 193 & 2.3036 & 2.3619 \\
\hline $\begin{array}{l}\text { Uni IM7 fibers } \\
\text { with epoxy ma- } \\
\text { trix }\end{array}$ & 1492 & 113 & 2.7139 & 2.7826 \\
\hline $\begin{array}{l}\text { Uni S-2 Glass } \\
\text { fibers with epoxy } \\
\text { (60\% FVF) }\end{array}$ & 2020 & 51.7 & 2.3322 & 2.3912 \\
\hline Aluminum & 2830 & 72 & 1.9705 & 2.0203 \\
\hline Steel titanium & 6450 & 75 & 1.3052 & 1.3383 \\
\hline $\begin{array}{l}\text { Nickel- } \\
\text { alloy }\end{array}$ & 210 & & \\
\hline
\end{tabular}

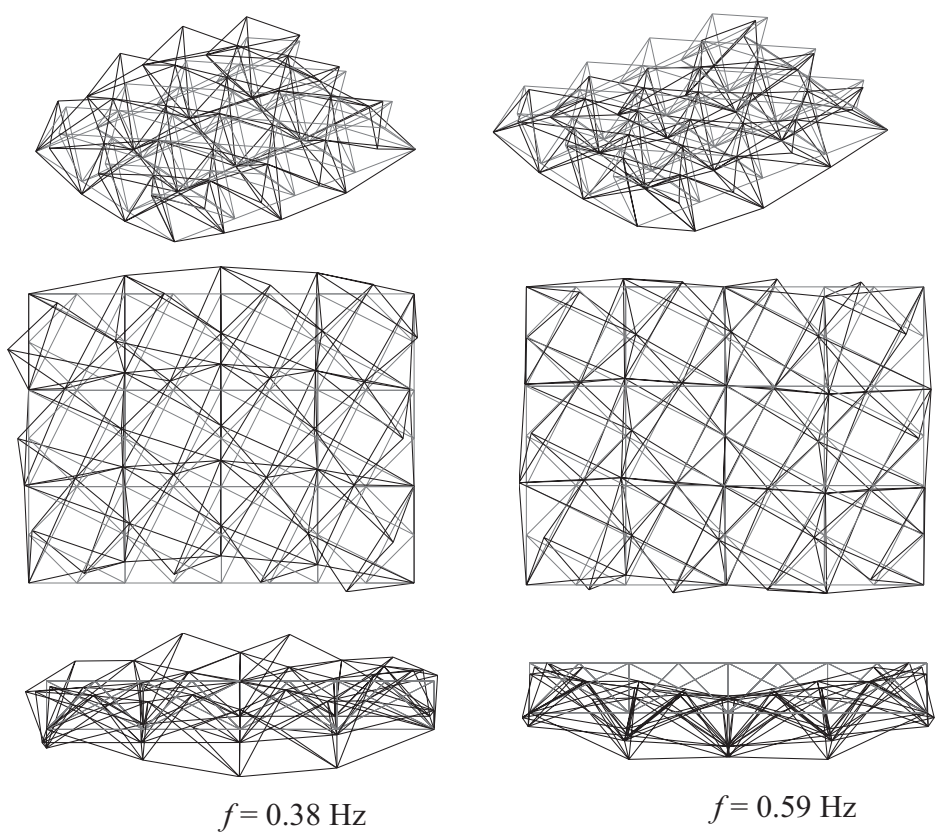

Figure 6. First two free vibration modes $P=1 \mathrm{kN}$. 

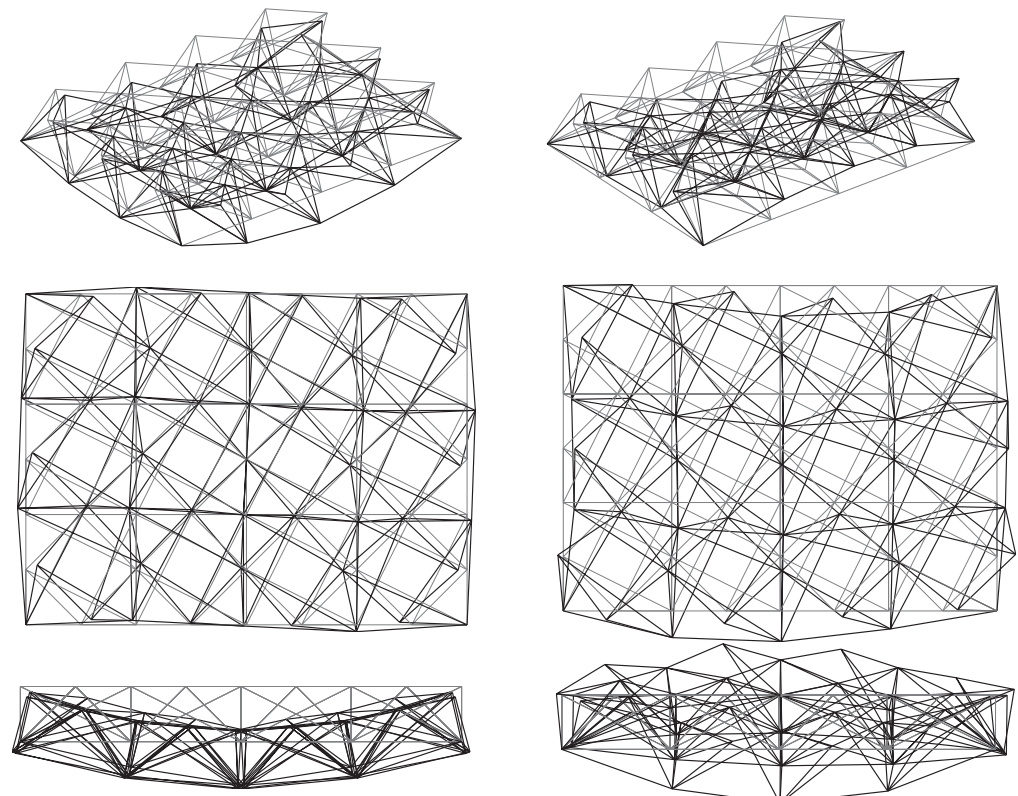

$f=1.30 \mathrm{~Hz}$

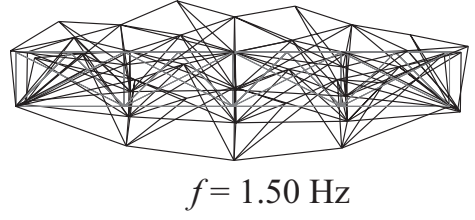

Figure 7. First two free vibration modes $P=100 \mathrm{kN}$.

\section{Conclusions}

An efficient method is presented for finding the optimum self-stress state in a tensegrity structure. Although a number of methods are presented, there is no research which connects the self-stress design to the level of pre-stress and the stiffness properties of the structure, e.g., the first natural frequency. The important aspect of this work is that all three steps connected to pre-stress are performed at the same stage. The method is applicable for design of the structure when natural frequencies are the major design criteria. In addition to that, a connection between GA as an optimization tool and FEM as a method for structural analysis is another a aspect of the current work.

\section{References}

[1] R. Motro, V. Raducanu, Tensegrity Systems, International Journal of Space Structures, 18(2), 77-84, 2003.

[2] J. Quirant, Self-stressed systems comprising elements with unilateral rigidity: selfstress states, mechanisms and tension setting, International Journal of Space Structures , 22(4), 203-214 2007.

[3] S. Pellegrino, Structural computations with the singular value decomposition of the equilibrium matrix, International Journal of Solids and Structures , 30 (21), 3025-3035, 1993.

[4] H. C. Tran, J. Lee, Initial self-stress design of tensegrity grid structures, Computers and Structures , 88 (9-10), 558-566, 2010. 
[5] X. Xu, Y. Luo, Force finding of tensegrity systems using simulated annealing algorithm, Journal of Structural Engineering , 136, 1027-1031, 2010.

[6] R. Connelly, Tensegrity structures: why are they stable?, Rigidity Theory and Applications, Kluwer Academic Publishers, Plenum Publishers, Dordrecht, New York , 47-54, 1999.

[7] M. Ohsaki, J. Zhang, Stability conditions of prestressed pin-jointed structures, International Journal of Non-Linear Mechanics , 41, 1109-1117, 2006.

[8] S. El-Lishani, H. Nooshin, P. Disney, Investigating the statical stability of pin-jointed structures using genetic algorithm, International Journal of Space Structures , 20 (1), 53-68, 2005.

[9] S. Guest, The stiffness of prestressed frameworks: A unifying approach, International Journal of Solids and Structures , 43, 3-4, 2006.

[10] A. Eriksson, Structural instability analyses based on generalised path-following, Computer Methods in Applied Mechanics and Engineering , 156 (1-4), 45-74, 1998.

[11] A. G. Tibert, S. Pellegrino, Review of form-finding methods for tensegrity structures, International Journal of Space Structures , 18 (4), 209-223, 2003.

[12] T. W. Murphey, Recent advances in gossamer spacecraft (Progress in Astronautics and Aeronautics), AIAA, 2006, Chapter 1.

[13] S. Dalil Safaei, A. Eriksson, G. Tibert, Application of flexibility analysis for design of tensegrity structures, in: Motro, R., ed, Structural Engineering World Congress (SEWC), 2011 .

[14] S. Dalil Safaei, G. Tibert, Design and analysis of tensegrity power lines, International Journal of Space Structures , 27, 2-3, 2012. 